\title{
Top Physics at CMS/LHC
}

\author{
Georgios Daskalakis ${ }^{1, a}$, On behalf of the CMS Collaboration \\ ${ }^{1}$ Institute of Nuclear and Particle Physics (INPP), NCSR Demokritos, Aghia Paraskevi, Greece
}

\begin{abstract}
Recent results on the inclusive and differential production cross sections of top-quark pair and single top-quark processes are presented, obtained using data from proton-proton collisions collected with the CMS detector at the LHC. The large centreof-mass energies available at LHC allow for the copious production of top-quark pairs in association with other final state particles at high transverse momentum. Measurements of such processes as well as of the top-quark mass and other properties will be discussed. The results are compared with the most up-to-date standard model theory predictions.
\end{abstract}

\section{Introduction}

Today, more than 20 years after the discovery of the top quark, particle physicists still investigate its properties by performing precision measurements. The top quark is the heaviest known elementary particle with a very short lifetime $\left(\tau_{\mathrm{t}}=1 / \Gamma_{\mathrm{t}} \sim 3.3 \times 10^{-25} \mathrm{~s}\right)$ [1]. It decays semi-weakly into a real $\mathrm{W}$ boson and a b quark before hadronization $\left(\tau_{\text {hadronization }} \sim 10^{-24} \mathrm{~s}\right)$ can occur. Due to its short lifetime, there is no sufficient time to depolarize its $\operatorname{spin}\left(\tau_{\text {spin decorrelation }} \sim 10^{-21} \mathrm{~s}\right)$ [2], so its spin information is transferred on to its decay products. Therefore, it is possible to measure observables that depend on the top quark spin, providing a unique environment for tests of the Standard Model (SM) and for searches for physics beyond the Standard Model (BSM). In addition, it is the only quark whose Yukawa coupling to the Higgs boson is of the order of unity so it is often speculated that it might play a special role in the mechanism of electroweak symmetry breaking. Its phenomenology provides a unique laboratory where our understanding of the strong interactions can be tested mainly through the top quark pair production. At the same time, weak interactions can be tested through the top quark decay and single top quark production. Top quarks are a major source of background for almost all searches for BSM physics at the LHC. Precise understanding of the top quark cross sections and kinematics is crucial before claiming new physics.

The final states for the leading pair-production process can be divided into three classes depending on the decay mode of the $\mathrm{W}$ bosons. The t⿱t events in which both, one or none $\mathrm{W}$ bosons decay hadronically are referred to as the all-jets, lepton+jets $(1+$ jets $)$ or dilepton channels, respectively. Their relative contributions, including hadronic corrections, are $45.7 \%, 43.8 \%$ and $10.5 \%$. Most of the analyses focus on final states with muons and electrons since the $\tau$ leptons are more difficult to reconstruct. In addition to the quarks resulting from the top-quark decays, extra radiation from quarks and gluons in the event can lead to extra jets in the final state.

ae-mail: Georgios.Daskalakis@cern.ch 


\section{Inclusive Top-quark pair and Single-top production cross sections}

In pp collisions, top quarks are produced dominantly in pairs through the processes $g g \rightarrow \mathrm{t} \overline{\mathrm{t}}$ and $\mathrm{q} \overline{\mathrm{q}} \rightarrow$ $\mathrm{t} \overline{\mathrm{t}}$, at leading order (LO) in quantum chromodynamics (QCD). At $\sqrt{s}=13 \mathrm{TeV}$, approximately $85.7 \%$ of the production cross section is from gluon-gluon fusion, with the remainder from $q \bar{q}$ annihilation.

The most precise CMS measurements of the $\mathrm{t} \overline{\mathrm{t}}$ production cross section at $\sqrt{s}=13 \mathrm{TeV}$ comes from the dilepton channel [3]. The measurement used data that correspond to an integrated luminosity of $2.2 \mathrm{fb}^{-1}$ analyzing events with one electron and one muon (e $\mu$ channel) and at least two jets, one of which is required to be identified as a b-jet. The measured cross section is $\sigma_{\mathrm{t} \overline{\mathrm{t}}}=793 \pm 8$ (stat) \pm 38 (syst) \pm 21 (lumi) $\mathrm{pb}$, corresponding to a total relative uncertainty of $5.6 \%$. The corresponding $\mathrm{SM}$ prediction for the $\overline{\mathrm{t}}$ production cross section is $\sigma_{\mathrm{t}}^{\mathrm{NNLO}+\mathrm{NNLL}}=$ $832 \pm 29($ scale $) \pm 35\left(\mathrm{PDF}+\alpha_{s}\right) \mathrm{pb}$, as calculated with the $\mathrm{TOP}++$ program [4] at next-to-next-toleading-order (NNLO) in perturbative $\mathrm{QCD}$, including soft-gluon resummation at next-to-next-toleading-log (NNLL) order [5], assuming $\mathrm{m}_{t}=172.5 \mathrm{GeV}$. The first uncertainty is an estimate of the effect of missing higher-order corrections and reflects uncertainties in the factorization and renormalization scales, while the second one is associated with possible choices of PDFs and the strong coupling constant $\left(\alpha_{s}\right)$. The measured cross section is therefore in agreement with the expectations from the SM. Further analyses in the 1+jets channel and the all-jets channel, yield consistent results.

In Fig. 1 (left) the summary of the cross section measurements of top pair production in CMS is presented in comparison with the theory calculation at NNLO with NNLL soft gluon resummation. The Tevatron measurements are also shown.

The associate production of $t \bar{t}$ along with an electroweak gauge boson ( $\mathrm{W}$ or $\mathrm{Z}$ ) have been also studied. The cross sections of these processes are typically much smaller than the $\mathrm{t} \overline{\mathrm{t}}$, yet can provide key information on the properties of the top quark. In these final states, according to the SM, the $\mathrm{W}$ boson is expected to be produced via initial state radiation, while the Z-boson can also be radiated from a final-state top-quark and hence provides sensitivity to the top-quark neutral current weak gauge coupling. Both $\sigma_{\overline{\mathrm{t}} \mathrm{W}}$ and $\sigma_{\mathrm{tt} Z}$ would be altered in a variety of new physics models that can be param-

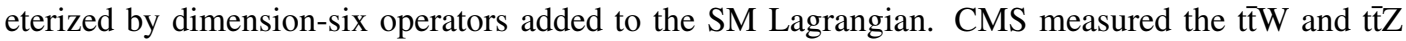
production cross sections using pp collision data at $\sqrt{s}=8 \mathrm{TeV}$ corresponding to an integrated luminosity of $19.5 \mathrm{fb}^{-1}$ [6]. Signatures from different decay modes of the top quark pair resulting in final states with two, three, and four leptons have been analyzed. The measured cross section in same-sign dilepton and three-lepton final states is $382_{-102}^{+117} \mathrm{fb}$, with an observed (expected) significance of 4.8 (3.5) standard deviations from the background-only hypothesis. Combining opposite-sign dilepton, three-lepton, and four-lepton channels, the t⿱亠乂 Z cross section is measured to be $242_{-55}^{+65} \mathrm{fb}$, an observation with a significance of 6.4 standard deviations from the background-only hypothesis, and in agreement with the SM expectation. The measured cross sections are used to constrain the vector and axial couplings of the $\mathrm{Z}$ boson to the top quark and to set bounds on the Wilson coefficients of five dimension-six operators that would affect the $\mathrm{t} \overline{\mathrm{t}} \mathrm{W}$ and $\mathrm{t} \overline{\mathrm{t}} \mathrm{Z}$ cross sections by parameterizing new physics. The measurements are compatible with the SM predictions. Recently, the cross section of $t \bar{t}$ production in association with a $\mathrm{Z}$ boson was measured using pp collisions at $\sqrt{s}=13 \mathrm{TeV}$ [7]. The data sample used corresponds to an integrated luminosity of $2.7 \mathrm{fb}^{-1}$. The measurement is performed in three- and four-lepton final states where the jet and b-jet multiplicities were exploited to enhance the signal over background ratio. The $\mathrm{t} \overline{\mathrm{Z}} \mathrm{Z}$ production cross section was measured to be $1065_{-313}^{+352}$ ( stat. )$_{-142}^{+168}$ ( sys. ) fb with a significance of 3.6 standard deviations from the background-only hypothesis.

The electroweak single top-quark production mechanisms, namely from $\mathrm{q}^{\prime} \rightarrow \mathrm{tb}, \mathrm{qb} \rightarrow \mathrm{q}^{\prime} \mathrm{t}$, mediated by virtual s-channel and t-channel $\mathrm{W}$ bosons, and tW-associated production, through bg $\rightarrow$ $\mathrm{tW}^{-}$, lead to smaller cross sections with respect to $\mathrm{t} \overline{\mathrm{t}}$ production. The $\mathrm{t}$-channel, while suppressed by the weak coupling with respect to the strong pair production, results in a sizable cross section at the 

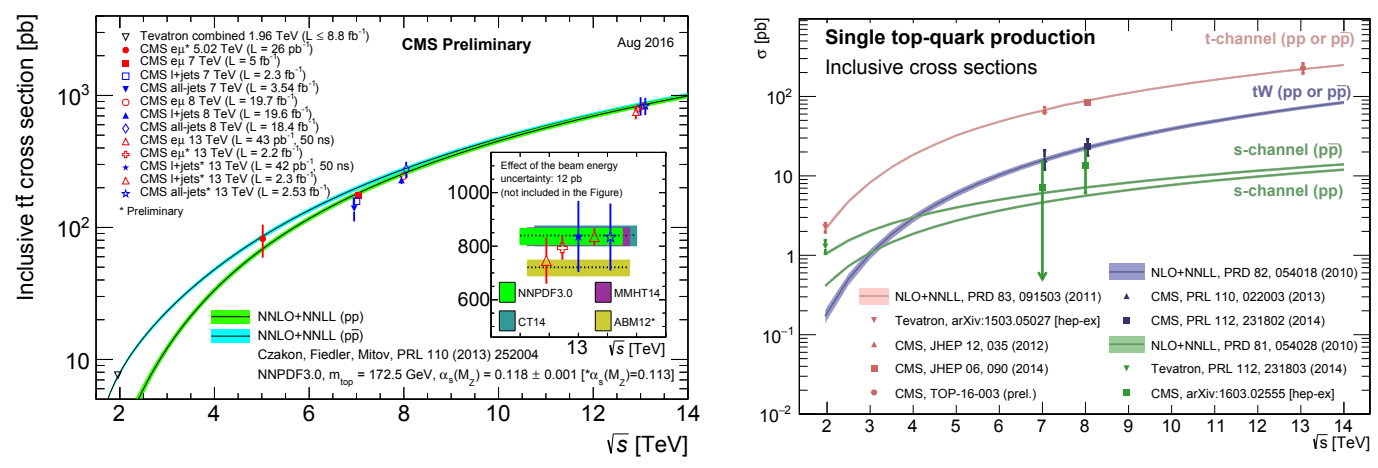

Figure 1. Left: Summary of the top quark pair cross section measurements from CMS, versus the centre-ofmass energy, in comparison with the theory calculation at NNLO+NNLL accuracy. Right: Summary of the single top cross section measurements from CMS, versus the centre-of-mass energy, in comparison with the theory calculation at NLO+NNLL accuracy. The Tevatron measurements are also shown.

LHC energies. The t- and s-channel cross sections of top and anti-top are not identical, due to the charge-asymmetric initial state. Measurements of the s- and t-channel production cross sections as well as the tW mechanism though the t-channel allow an extraction of the CKM matrix element $\mathrm{V}_{t b}$.

A recent measurement of the t-channel single top quark production cross section was performed by CMS at $\sqrt{s}=13 \mathrm{TeV}$ [8], analyzing data that correspond to an integrated luminosity of $2.3 \mathrm{fb}^{-1}$. The muon+jets signature was explored and several kinematic variables were combined into one multivariate discriminator optimized to separate signal from background events. A fit to the distribution of the discriminating variable yields cross sections of :

$$
\begin{aligned}
& \left.\sigma_{t-c h ., t}=141.5 \pm 6.7 \text { (stat. }\right) \pm 9.4(\text { exp. })_{-19.6}^{+19.3} \text { (theo.) } \pm 3.8 \text { (lumi.) } \mathrm{pb} \text { and } \\
& \left.\left.\sigma_{t-c h ., \bar{t}}=81.0 \pm 6.2 \text { (stat. }\right) \pm 8.1 \text { (exp. }\right)_{-10.9}^{+10.9} \text { (theo.) } \pm 2.2 \text { (lumi.) pb }
\end{aligned}
$$

for the production of single top quarks and single top anti-quarks, respectively. The cross section ratio between top quark and anti-quark production is measured to be: $\mathrm{R}_{t-c h}=1.75 \pm 0.16$ (stat.) \pm 0.21 (syst.). A comparison between the measured ratio and the prediction of different PDF sets is shown on the left plot of Fig. 2. In case of MSTW2008NLO [9] and of NNPDF [10] the fixed four-flavour scheme (4FS) PDFs are used together with the POWHEG 4FS calculation. The POWHEG calculation in the five-flavour scheme (5FS) is used for all other PDFs, as they are variable flavor scheme PDFs.

Assuming $\left|\mathrm{V}_{t b}\right|$ much greater than $\left|\mathrm{V}_{t d}\right|,\left|\mathrm{V}_{t s}\right|$ the cross sections for single top production are proportional to $\left|\mathrm{V}_{t b}\right|^{2}$. From the measured inclusive cross section, $\sigma_{t-c h}=$ $227.8 \pm 9.1$ (stat.) \pm 14.0 (exp.) ${ }_{-27.7}^{+28.7}$ (theo.) \pm 6.2 (lumi.) $\mathrm{pb}$ and its predicted value, the CKM matrix element $\left|\mathrm{V}_{t b}\right|$ is calculated to be $\left|\mathrm{V}_{t b}\right|=1.02 \pm 0.07$ (exp.) \pm 0.02 (theo.). All results are in agreement with the SM predictions.

CMS performed a search for single top quark production in the s-channel in decay modes of the top quark containing a muon or an electron in the final state [11]. The signal is extracted through a maximum-likelihood fit to the distribution of a multivariate discriminant defined to separate the expected signal contribution from background processes. The analysis uses data corresponding to integrated luminosities of $5.1 \mathrm{fb}^{-1}$ at $7 \mathrm{TeV}$ and $19.7 \mathrm{fb}^{-1}$ at $8 \mathrm{TeV}$, respectively. The measured cross sections of $7.1 \pm 8.1 \mathrm{pb}$ at $7 \mathrm{TeV}$ and $13.4 \pm 7.3 \mathrm{pb}$ at $8 \mathrm{TeV}$ result in a best fit value of $2.0 \pm 0.9$ for the combined ratio of the measured and expected values. The signal significance is 2.5 standard 

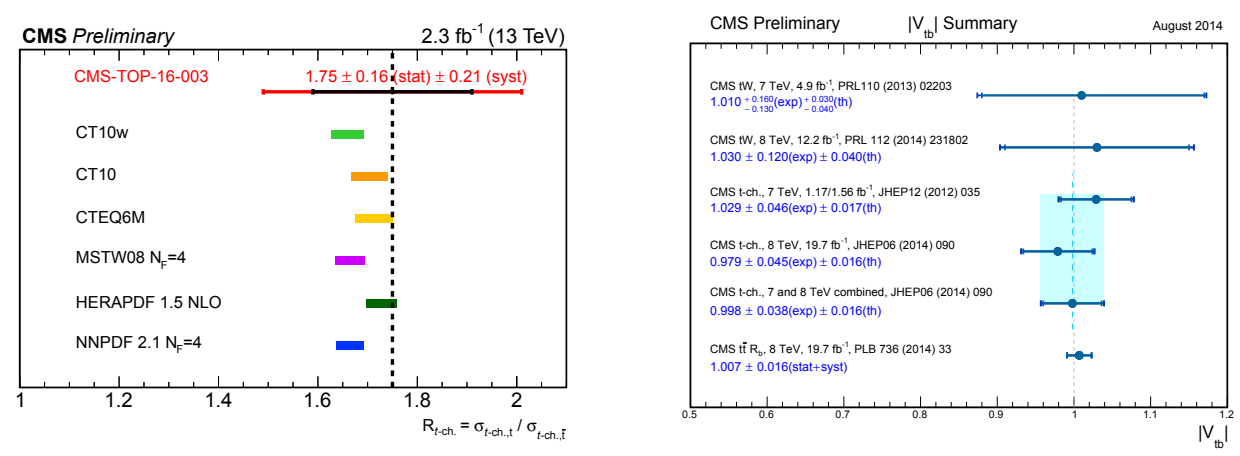

Figure 2. Left: Comparison of the measured $\mathrm{R}_{t-c h .}=\sigma_{t-c h, t} / \sigma_{t-c h ., \bar{t}}$ with the prediction obtained using different PDF sets. The nominal value for the top quark mass is $173.0 \mathrm{GeV}$. Error bars for the different PDF sets include the statistical uncertainty, the uncertainty on the factorization and renormalization scales, and the uncertainty on the top quark mass. Right: Summary of $\left|\mathrm{V}_{t b}\right|$ measurements in CMS.

deviations, and the upper limit on the rate relative to the SM expectation is 4.7 at $95 \%$ confidence level.

The $\mathrm{tW}$ process is of interest because it probes the $\mathrm{tWb}$ vertex in a different kinematic region than $\mathrm{s}$ - and t-channel production. The definition of $\mathrm{tW}$ production in perturbative QCD at NLO mixes with top-quark pair production so special treatment is necessary in order to disentangle the two processes $[12,13]$. At measurement level the selection cuts are defined such that the interference effects are small. The associated production of a single top quark and a $\mathrm{W}$ boson was measured in CMS by using a data set of $12.2 \mathrm{fb}^{-1}$ of pp collisions at $\sqrt{s}=8 \mathrm{TeV}$ [14]. Events with two leptons and a jet originating from a $b$ quark are selected. A multivariate analysis based on kinematic and topological properties is used to separate the signal from the dominant $t \bar{t}$ background. An excess consistent with the signal hypothesis is observed, with a significance which corresponds to 6.1 standard deviations above a background-only hypothesis. The measured production cross section is $23.4 \pm 5.4 \mathrm{pb}$, in agreement with the SM prediction.

In Fig. 1 (right) a summary of single top cross-section measurements at CMS are presented as a function of the center-of-mass energy in comparison with the theory calculation at NLO+NNLL accuracy. The Tevatron measurements are also shown. All cross-section measurements are very well described by the theory calculations within their uncertainty. In Fig. 2 (right) a summary of $\mathrm{V}_{t b}$ determinations by CMS is presented.

\section{Differential cross sections}

Thanks to the large available luminosity, CMS performed differential cross-section measurements in $\mathrm{t} \overline{\mathrm{t}}$ production. Such measurements are crucial, as they allow even more stringent tests of perturbative QCD as description of the production mechanism and enhance the sensitivity to possible new physics contributions, especially now that NNLO predictions for the main differential observables in $\mathrm{t} \overline{\mathrm{t}}$ production have become available [15]. Furthermore, such measurements reduce the uncertainty in the description of $t \bar{t}$ final state as background in Higgs physics, searches for rare processes, or BSM physics.

Differential cross-sections are typically measured by applying a kinematic reconstruction on the selected candidate events and subsequent unfolding the obtained event counts in bins of kinematic 

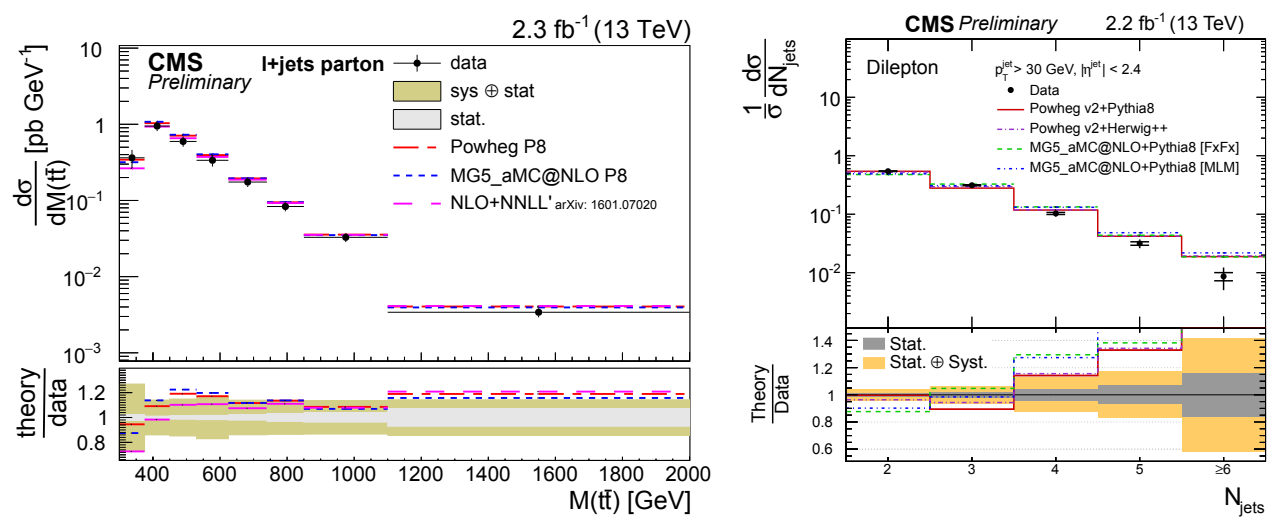

Figure 3. Left: Differential cross sections at parton level as a function of $\mathrm{M}_{\mathrm{tt}}$ compared to the $\mathrm{SM}$ predictions. The measurements are performed in the lepton+jets decay channel with an electron or a muon in the final state. Right: Normalized differential tī production cross section as a function of the jet multiplicity. The inner (outer) error bars indicate the statistical (combined statistical and systematic) uncertainty. The measurements have been performed in dilepton decay channels.

distributions in order to correct for detector resolution effects, acceptance and migration effects. By using pp collisions at $13 \mathrm{TeV}$ with $2.3 \mathrm{fb}^{-1}$ of integrated luminosity CMS measured the differential $\overline{\mathrm{t}}$ cross section as a function of various kinematic variables of the t⿱t system as well as jet multiplicities. The measurements are performed in the lepton+jets decay channel with an electron or a muon in the final state [16]. The differential cross sections are presented at particle level, within a phase space close to the experimental acceptance, and at parton level in the full phase space. The measurement at parton level is dominated by uncertainties in the parton shower and hadronization model. The dependency on these theoretical models is reduced for the particle level measurement, for which the experimental uncertainties of jet energy calibration and $\mathrm{b}$ tagging efficiency are the most dominant. The results are compared to several SM predictions of approximate NNNLO [17, 18] and NLO+NNLL' [19] calculations as shown in the left plot of Fig. 3. All calculations are compatible with the measured results. Similar measurements have been performed in dilepton decay channels at $13 \mathrm{TeV}$ [20] using data that correspond to an integrated luminosity of $2.2 \mathrm{fb}^{-1}$. The results are compared with several perturbative QCD calculations as shown in the right plot of Fig. 3 and are found to be broadly in agreement with the predictions.

Differential cross-section measurements were also performed by CMS in single-top t-channel production. Such measurements are extremely useful as they test our understanding of both QCD and EW top-quark interactions. Using pp collisions at a centre-of-mass energy of $13 \mathrm{TeV}$ and analyzing data that corresponded to an integrated luminosity of $2.3 \mathrm{fb}^{-1}$ the differential cross sections were measured as functions of the transverse momentum and the absolute value of the rapidity of the top quark [21]. A maximum-likelihood fit to a multivariate discriminator is used to infer the signal and background fractions from the data. Unfolding to parton level is performed in this case. The measured cross sections are compared with theoretical predictions to next-to-leading order matched with parton showering as implemented in Monte Carlo generators. General agreement is found within uncertainties as shown in Fig. 4. 

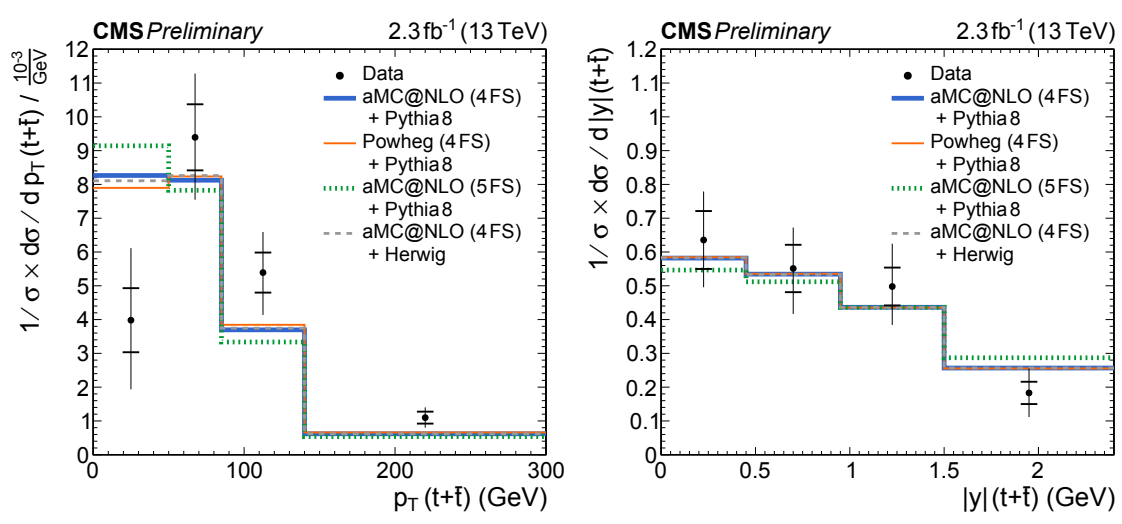

Figure 4. Left: Measured differential cross section of t-channel single top quark production as a function of the top quark transverse momentum. Right: Measured differential cross section of t-channel single top quark production as a function of the top quark rapidity. Horizontal ticks on the error bars indicate the statistical uncertainty and vertical bars indicate the total uncertainty per bin.

\section{Top-quark mass measurements}

The mass of the top quark $\left(\mathrm{m}_{t}\right)$ is one of the fundamental parameters of the SM. With the discovery of a Higgs boson at the LHC with a mass of about $126 \mathrm{GeV}[22,23]$, the precision measurement of the top-quark mass takes a central role in the question of the stability of the electroweak vacuum. Recent NNLO calculations $[24,25]$ together with the central values of the Higgs and top-quark masses put the electroweak vacuum in the metastable region. The uncertainty is dominated by the precision of the top-quark mass measurement and its interpretation as the pole mass.

CMS has measured the top quark mass using pp data at $\sqrt{s}=8 \mathrm{TeV}$ corresponding to a luminosity of $19.7 \mathrm{fb}^{-1}$ [26]. In the measurement all decay channels were used. The measurements use analysis techniques in which either $\mathrm{m}_{t}$ alone is determined or $\mathrm{m}_{t}$ and the overall jet energy scale factor are determined simultaneously. When the results from the $\sqrt{s}=8 \mathrm{TeV}$ analysis are combined with the published CMS results at $\sqrt{s}=7 \mathrm{TeV}$, a top quark mass measurement of $172.44 \pm 0.13$ (stat.) \pm 0.47 (syst.) $\mathrm{GeV}$ is achieved. Given the precision of the experimental results, a detailed understanding of the relationship between the measurements and the value of $\mathrm{m}_{t}$ in different theoretical schemes is needed. Current indications are that the present measurements based on the kinematic reconstruction of the top quark mass correspond approximately to the pole ("on-shell") mass to within a precision of about $1 \mathrm{GeV}$ [27]. A summary of the CMS $\mathrm{m}_{t}$ measurements and their combination is shown in the left plot of Fig. 5. The top-quark mass can be extracted by using alternative techniques or observables that might have sensitivity on the $\mathrm{m}_{t}$ like the normalized invariant mass distribution of the $\bar{t}+$ jet system, the peak position of the energy distribution of $b$ jets produced from top-quark decays and several others. A long list of alternative measurements is shown in the right hand-side plot of Fig. 5 together with the references to the corresponding documentation.

\section{Top polarization, $\mathrm{t} \overline{\mathrm{t}}$ spin correlations and charge asymmetry}

One of the unique features of the top quark is that it decays before its spin can be flipped by the strong interaction. Thus the top-quark polarization is directly observable via the angular distribution of its 

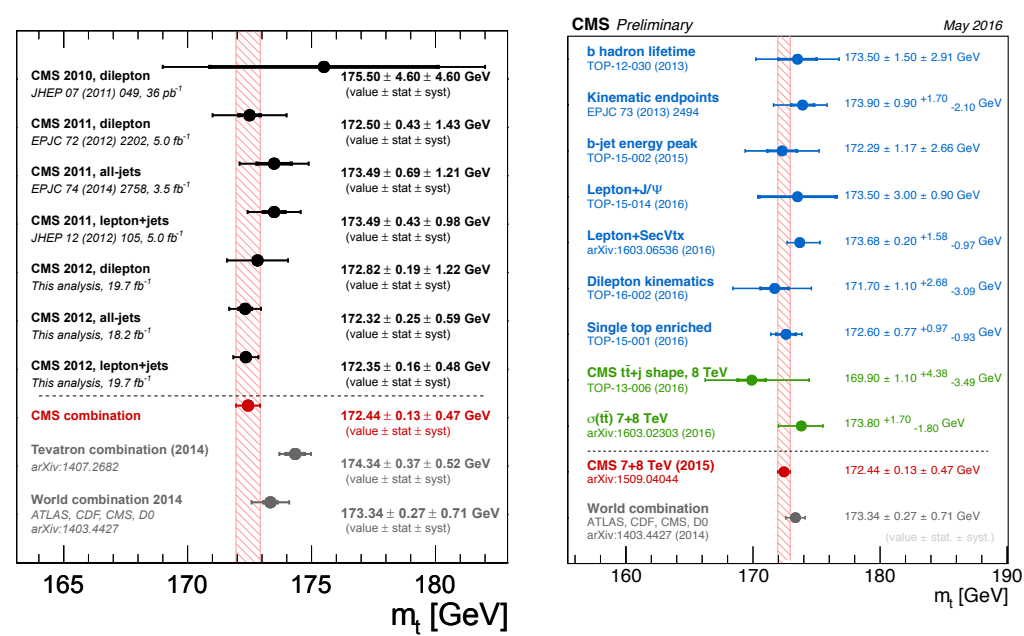

Figure 5. Left: Summary of the CMS $\mathrm{m}_{t}$ measurements and their combination. The thick error bars show the statistical uncertainty and the thin error bars show the total uncertainty. Also shown are the current Tevatron [28] and world average [29] combinations. Right: Summary of Run-I CMS alternative $\mathrm{m}_{t}$ measurements.

decay products. In the SM, top quarks from pair production have only a small net polarization arising from electroweak corrections to the QCD-dominated production process, but the pairs have significant spin correlations [30]. Hence, it is possible to define and measure observables sensitive to the topquark spin and its production mechanism. For low $t \bar{t}$ invariant masses, the production is dominated by the fusion of pairs of gluons with the same helicities, resulting in the creation of top quark pairs with antiparallel spins in the $t \bar{t}$ center-of-mass frame. For larger $t \bar{t}$ invariant masses, the dominant production is via the fusion of gluons with opposite helicities, resulting in t $\bar{t}$ pairs with parallel spins. For BSM models, couplings of the top quark to new particles can alter both the top quark polarization and the strength of the spin correlations in the $\bar{t} \bar{t}$ system [30-33].

CMS has measured the top quark-antiquark spin correlations and the top quark polarization for $\mathrm{t}^{\mathrm{t}}$ pairs produced in pp collisions at $\sqrt{s}=8 \mathrm{TeV}$ [34]. The data correspond to an integrated luminosity of $19.5 \mathrm{fb}^{-1}$. The measurements are performed using events with two oppositely charged leptons (electrons or muons) and two or more jets, where at least one of the jets is identified as originating from a bottom quark. The spin correlations and polarization are measured from the angular distributions of the two selected leptons, both inclusively and differentially, with respect to the invariant mass, rapidity, and transverse momentum of the $\bar{t} \bar{t}$ system. The measurements are unfolded to the parton level and found to be in agreement with predictions of the SM as can be seen in Fig. 6.

The $t \bar{t}$ charge asymmetry is sensitive to BSM contributions that might affect the $t \bar{t}$ production process. The dominant $t \bar{t}$ production mechanism is the charge-symmetric gluon-gluon fusion. For the sub-dominant qq production mechanism, the symmetric pp collision does not define a forward and backward direction. Instead, the larger average momentum fraction of the valence quarks leads to an excess of top quarks produced in the forward and backward directions, while the top antiquarks are produced more centrally. This makes the difference in the absolute values of the rapidities of the top quark and anti-quark, $\Delta|\mathrm{y}|=\left|\mathrm{y}_{t}\right|-\left|\mathrm{y}_{\bar{t}}\right|$, a suitable observable to measure the $\mathrm{t} \overline{\mathrm{t}}$ charge asymmetry at the LHC. The charge asymmetry, $A_{C}$, is defined as $A_{C}=\left(\mathrm{N}^{+}-\mathrm{N}^{-}\right) /\left(\mathrm{N}^{+}+\mathrm{N}^{-}\right)$, where $\mathrm{N}^{+}$and $\mathrm{N}^{-}$ represent the number of events with positive and negative values of $\Delta|\mathrm{y}|$, respectively. To shed light 

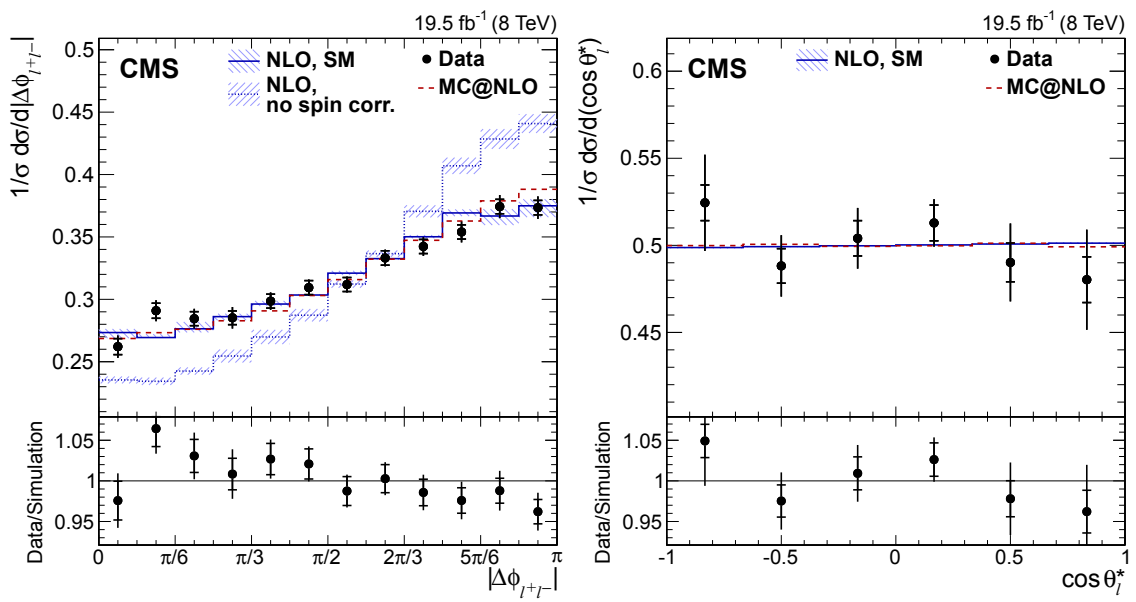

Figure 6. Left: Normalized differential cross section as a function of $\left|\Delta \phi_{l^{+}, l^{-}}\right|$. Right: Normalized differential cross section as a function of $\cos \theta_{l}^{*}$. The plots are shown data (points); parton-level predictions from MC@NLO [35, 36] (dashed histograms); and theoretical predictions at NLO [30, 37] with (SM) and without (no spin corr.) spin correlations (solid and dotted histograms, respectively). For the $\cos \theta_{l}^{*}$ distribution, CP conservation is assumed in the combination of the $\cos \theta_{l}^{*}$ measurements from positively and negatively charged leptons. The ratio of the data to the MC@ NLO prediction is shown in the lower panels. The inner and outer vertical bars on the data points represent the statistical and total uncertainties, respectively.

on the possible existence and the nature of new physics contributions, it is crucial to measure not only the inclusive asymmetry but also $A_{C}$ as a function of variables magnifying the $\bar{t}$ charge asymmetry.

CMS has measured the $\bar{t} \bar{t}$ charge asymmetry at $8 \mathrm{TeV}$ with an integrated luminosity of 19.7 $\mathrm{fb}^{-1}$ [38]. Events are selected if they contain one electron or muon and four or more jets, at least one of which is identified as originating from the hadronization of a bottom quark. The measurement is based on the reconstructed four-momenta of the $t$ and $\bar{t}$ quarks in each event. The observed distributions are then corrected for background contributions, resolution, and selection efficiency. Acceptance corrections are applied, correcting either to the fiducial phase space or to the full phase space. The inclusive charge asymmetry is found to be $0.0010 \pm 0.0068$ (stat) \pm 0.0037 (syst). Measurements were also performed in a reduced fiducial phase space of top quark pair production, with an integrated result of $-0.0035 \pm 0.0072$ (stat) \pm 0.0031 (syst). All measurements are consistent within two standard deviations with zero asymmetry as well as with the predictions of the SM.

A summary of the charge asymmetry measurements on ATLAS and CMS at $8 \mathrm{TeV}$ is shown on the left plot of figure 7. The plot shows both the inclusive measurements and the measurement using boosted events, compared to the respective theory predictions. The right hand-side plot of figure 7 shows the corrected asymmetry as a function of $\mathrm{m}_{\mathrm{t} \mathfrak{\mathrm { t }}}$. The results correspond to the full phase space. The measured values are compared to NLO predictions for the SM based on calculations by Kühn and Rodrigo (K\&R) [39] and Bernreuther and $\mathrm{Si}(\mathrm{B} \& \mathrm{~S})$ [37, 40], as well as to the predictions of a model featuring an effective axial-vector coupling of the gluon (EAG) [41, 42]. The inner bars indicate the statistical uncertainties, while the outer bars represent the statistical and systematic uncertainties added in quadrature. The differential measurements show a good agreement with the SM predictions. For the benchmark model involving an effective axial-vector coupling of the gluon, the measurement at high $\mathrm{m}_{\mathrm{t}}$ excludes new physics scales below $1.5 \mathrm{TeV}$ at the $95 \%$ confidence level. 

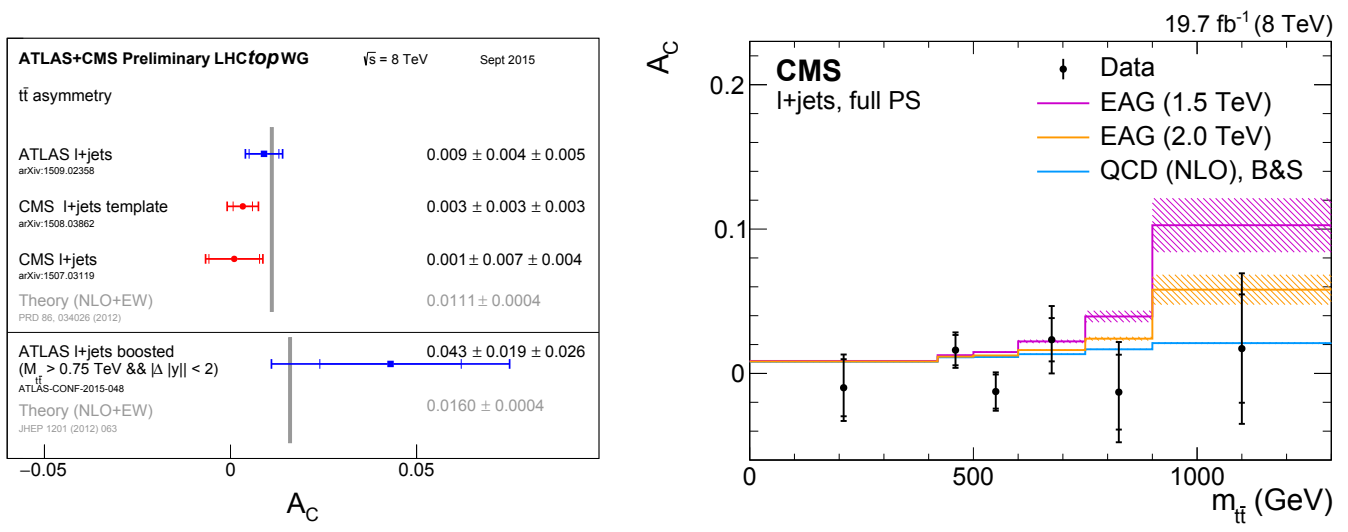

Figure 7. Left: Summary of the charge asymmetry measurements on ATLAS and CMS at $8 \mathrm{TeV}$ showing both the inclusive measurements and the measurement using boosted events, compared to the respective theory predictions. The uncertainty on the theory predictions is shown but is very small. Right: Corrected asymmetry as a function of $\mathrm{m}_{\mathrm{t}}$. The results correspond to the full phase space. The measured values are compared to NLO predictions for the SM, as well as to the predictions of a model featuring an effective axial-vector coupling of the gluon (EAG). The inner bars indicate the statistical uncertainties, while the outer bars represent the statistical and systematic uncertainties added in quadrature.

\section{Summary}

Top-quark physics at hadron colliders has developed into precision physics. Various properties of the top quark have been measured with high precision. Up to now, all measurements are consistent with the SM predictions and allow stringent tests of the underlying production mechanisms by strong and weak interactions. Given the large event samples available at the CMS experiment, top-quark properties has been determined both in $t \bar{t}$ as well as in electroweak single top-quark production. Furthermore, the $\bar{t} \bar{t}$ associated production with heavy bosons provides further information on the top-quark electroweak couplings. At the same time various models of physics beyond the SM involving top-quark production are being constrained. As the first results from LHC Run-II at a higher center-of-mass energy and much higher luminosity starting to be released, top-quark physics has the potential to shed light on open questions and new aspects of physics at the TeV scale.

\section{References}

[1] D0 Collaboration, Phys. Rev. D 85, 091104 (2012)

[2] G. Mahlon and S. J. Park, Phys. Rev. D 81, 074024 (2010)

[3] CMS Collaboration, CMS-PAS-TOP-16-005, CERN, Geneva (2016)

[4] M. Czakon and A. Mitov,Computer Physics Communications, 185, 2930 (2014)

[5] M. Czakon, P. Fiedler and A. Mitov, Phys. Lett. B 110, 252004 (2013)

[6] CMS Collaboration, J. High Energy Phys. 01 (2016) 096

[7] CMS Collaboration, CMS-PAS-TOP-16-009, CERN, Geneva (2016)

[8] CMS Collaboration, CMS-PAS-TOP-16-003, CERN, Geneva (2016)

[9] A. Martin, W. Stirling, and G. Watt, Eur. Phys. J. C 63, 189 (2009) 
[10] R. Ball, V. Bertone, F. Cerutti et al., Nucl. Phys. B 855, (2012) 608

[11] CMS Collaboration, J. High Energy Phys. 09 (2016) 027

[12] S. Frixione, E. Laenen, P. Motylinski et al., J. High Energy Phys. 07, (2008) 029

[13] T. M. P. Tait, Phys. Rev. D 61, (1999) 034001

[14] CMS Collaboration, Phys. Rev. Lett. 112, 231802 (2014)

[15] M. Czakon, D. Heymes, and A. Mitov, Phys. Rev. Lett. 116, 082003 (2016)

[16] CMS Collaboration, CMS-PAS-TOP-16-008, CERN, Geneva (2016)

[17] N. Kidonakis, Phys. Rev. D 90, 014006 (2014)

[18] N. Kidonakis, Phys. Rev. D 91, 031501 (2015)

[19] B. Pecjak, D. Scott, X. Wang, and L. L. Yang, Phys. Rev. Lett. 116, 202001 (2016)

[20] CMS Collaboration, CMS-PAS-TOP-16-011, CERN, Geneva (2016)

[21] CMS Collaboration, CMS-PAS-TOP-16-004, CERN, Geneva (2016)

[22] ATLAS Collaboration, Phys. Lett. B 716, 1 (2012)

[23] CMS Collaboration, Phys. Lett. B 716, 30 (2012)

[24] G. Degrassi, et al., J. High Energy Phys. 08, 98 (2012)

[25] A. V. Bednyakov, et al., Phys. Rev. Lett. 115, 201802 (2015)

[26] CMS Collaboration, Phys. Rev. D 93, 072004 (2016)

[27] A. Buckley, et al., Phys. Rep. 504, 145 (2011)

[28] The Tevatron EWK Working Group, CDF and D0 Collaborations, arXiv:1407.2682

[29] ATLAS, CDF, CMS and D0 Collaborations, arXiv:1403.4427

[30] W. Bernreuther and Z.-G. Si, Phys. Lett. B 725, 115 (2013)

[31] D. Krohn, T. Liu, J. Shelton, and L.-T. Wang, Phys. Rev. D 84, 074034 (2011)

[32] S. Fajfer, J. F. Kamenik, and B. Melic, J. High Energy Phys. 08 (2012) 114

[33] J. A. Aguilar-Saavedra and M. Perez-Victoria, J. Phys. Conf. Ser. 447, 012015 (2013)

[34] CMS Collaboration, Phys. Rev. D 93, 052007 (2016)

[35] S. Frixione and B. R. Webber, J. High Energy Phys. 06, 029 (2002)

[36] S. Frixione, P. Nason, and B. R. Webber, J. High Energy Phys. 08, 007 (2003)

[37] W. Bernreuther and Z.-G. Si, Phys. Lett. B 837, 90 (2010)

[38] CMS Collaboration, Phys. Lett. B 757, 154 (2016)

[39] J.H. Kühn, G. Rodrigo, J. High Energy Phys. 01, 063 (2012)

[40] W. Bernreuther, Z.-G. Si, Phys. Rev. D 86, 034026 (2012)

[41] E. Gabrielli, M. Raidal, A. Racioppi, Phys.Rev. D 85, 074021 (2012)

[42] E. Gabrielli, M. Raidal, Phys. Rev. D 84, 054017 (2011) 